Hinshelwood-nothing to inspire the research workers in chemical kinetics, to draw young people into the subject, or to give succour to those peris outside paradise, the workers in heterogeneous catalysis. This is, I fear, a rather dull book: a Moskvich rather than a Lotus Elan. D. D. ELEY

\section{Turbulent plasmas}

Plasma Astrophysics. By S. A. Kaplan and V. N. Tsytovich. Translated by D.ter Haar. Pp. xiii + 302. (International Series of Monographs in Natural Philosophy.) (Pergamon: Oxford and New York, November 1973.) £9.50.

THIS book follows on naturally from An Introduction to the Theory of Turbulent Plasmas by Tsytovich, bringing together references to many of the papers published by Russian workers in the last few years. In this work the authors are concerned primarily with radiation and particle acceleration by turbulent plasmas in the sun, pulsars, nuclei of galaxies, radio galaxies and quasars. The authors restrict their attention to weak turbulence where the energy density of plasma waves is much less than the plasma thermal energy density. They assert that strong turbulence decays on a time scale which is short compared with astrophysical times leaving the plasma in a state of weak turbulence. Recently discovered 'spicons' in laboratory plasmas with wave energy densities large in comparison with the thermal energy density may eventually cast some doubt on the universal applicability of the weak turbulence assumption to astrophysical plasmas. Within the weak turbulence assumption the authors discuss three types of turbulence: 'heating turbulence', where the energy of plasma waves is dissipated through electron-ion collisions and Landau damping; 'acceleration plasma turbulence', where the wave energy is lost in accelerating particles; and 'radiation plasma turbulence' where low frequency plasma wave energy is lost by the generation of high frequency electromagnetic waves produced by the interaction of plasma with particles.

The book is divided into four chapters: the first reviews, with liberal reference to the literature, laboratory and theoretical developments in plasma turbulence. The second chapter deals with sporadic solar emission, for example, how electromagnetic waves may be generated by ion-sound turbulence. The third discusses how turbulent processes can lead to observable radiation in for example radio galaxies and quasars, and the book ends with a discussion of pulsar emission.

The authors consider that the most important causes of plasma turbulence, at least in a narrow frequency range are: beam, ion drift, loss-cone and anisotropy plasma instabilities. To these one should probably now add parametric instabilities occurring in the wave zones of pulsars. It is not yet clear whether refractive index effects, incurred by requiring a dense plasma to act as a vehicle for the turbulence, will inhibit radiative processes involving relativistic particles. The turbulent reactor production of a cosmic-ray ion power low spectrum meets with some difficulty if located in pulsars. The authors prefer to have the source in the nucleus of the galaxy; the implication for isotropy is not clear from their arguments.

The book is clearly written and the translator is obviously familiar with the scientific content of the work. The book is a very important addition to the astrophysicist's library; the new graduate student might however consider reading a more elementary text before attempting it. P. STEWART

\section{Classifying vegetation}

Ordination and Classification of Communities. Edited by R. H. Whittaker. Pp. $x+738$. (Handbook of Vegetation Science, Vol. 5.) (Junk: The Hague, 1973.) Dfl. 160.

A DECADE ago the production of a volume such as this would not have been possible, but through the agency of the International Society for Plant Geography and Ecology symposia, the different approaches to vegetation, each with its own recondite peculiarities have been interchanged, discussed and dissected. But this is only half the story. To coax and cajole nineteen eminent practitioners from eight countries to contribute to the tome is no mean feat, and the praise for the commonplace drudgery and exasperating mechanics of coordination of the contributions falls firmly upon the energetic frame of Robert Whittaker. There can be no doubt that the dynamic personality of this man and the depth of scientific respect which he commands made the project a viable one, and the patient understanding provided by the publishers helped to ensure a production of the highest quality.

The book is divided naturally into three sections-direct gradient analysis, indirect gradient analysis and classification. The first section contains five papers, four of which have Whittaker as senior author, and a fifth written by Sobolev and Utekhin of the Soviet Academy of Sciences, Moscow. Much of Whittaker's contribution seems to be a new presentation of his earlier works, particularly his 1967 Biological Review paper, but as a part of a general overview of methodology, its presence is vital. The two Russian authors provide a refreshing interpretation of the much abused Ramensky continuum concept which makes possible a far deeper insight into this approach than has been previously possible.

The second section contains typically thorough and informative papers on sample similarity and species correlation by Goodall, factor analysis by Dagnelie and ordination by resemblance matrices contributed by Orloci. The methods of comparative ordination evolved by the Wisconsin school some twenty years ago are re-examined by Cottam, Goff and Whittaker and, like the paper on matrix and plexus techniques by McIntosh will win many plaudits for its simplicity and clarity of presentation. But pride of place in this section must be given to the invaluable paper of Whittaker and Gauch which provides a realistic and erudite evaluation of ordination techniques using such criteria as efficiency, relative freedom from distortion of sample relationship and computational expenditure.

Whittaker is also responsible for two chapters in section 3--one, his familiar enumeration of the bases of vegetation classification and the different traditions which have evolved, the other a short discussion on the nature of dominance types which neatly complements the paper on the physiognomic approach by Beard. To many European phytosociologists, chapters 15 to 18 and 20 will prove to be invaluable, if not the highlight of the book. They comprise papers written by practitioners from various European countries who are fully conversant with the intricacies of and interpretations to be derived from methods which they themselves have used extensively. In this respect, the contributions of Frey, Alexsandrova and Trass and Malmer on the Finnish, Russian and Scandinavian/ Baltic approaches respectively help to fill a comparatively large gap in readily available literature in the English language. The synusial approach to classification is admirably dealt with by Barkman who covers a wide range of applications from epiphyte societies to aquatic vegetation types. In their exposition of the Braun-Blanquet methods, Westhoff and van der Maarel provide what is perhaps the best insight into the methodology in all its facets that has yet been written in English. A contribution on numerical taxonomy from Goodall completes this comprehensive section and the book.

There can be no doubt that this work will become one of the ecological classics. It is already my vade mecum $\begin{array}{ll}\text { vegetatio. } & \text { D. W. SHIMWELL }\end{array}$ 